\title{
Facing Coronavirus Disease 2019 Pandemic Situation in Pharmaceutical Companies: Challenges and Solutions
}

\author{
Seyed Hamid Falaki ${ }^{1,2}$, Maryam Safaeikoshkak ${ }^{3}$, Jaber Abedi ${ }^{4}$ and Milad Ahmadi Marzaleh ${ }^{5, *}$ \\ 1 MSc in Management of Health, Safety and Environment, School of Health and Paramedical, Zanjan University of Medical Sciences, Zanjan, Iran \\ 2 Head of HSE Department, Tehran Chemie Pharmaceutical Co., Tehran, Iran \\ ${ }^{3}$ BSc in Occupational Health Engineer, School of Public Health, Tehran University of Medical Sciences, Tehran, Iran \\ 4 MSc Student of Health, Safety and Environment Management, School of Public Health, Zanjan University of Medical Sciences, Zanjan, Iran \\ 5 Department of Health in Disasters and Emergencies, School of Management and Medical Informatics, Shiraz University of Medical Sciences, Shiraz, Iran
}

* Corresponding author: Milad Ahmadi Marzaleh, Department of Health in Disasters and Emergencies, School of Management and Medical Informatics, Shiraz University of Medical Sciences, Shiraz, Iran. Tel: +987132340774; Fax: +987132340039; Email: miladahmadimarzaleh@yahoo.com

Received 2021 February 10; Revised 2021 June 12; Accepted 2021 August 18.

Keywords: COVID-19, Health, New coronavirus, Pandemic, Pharmaceutics, Pharmaceutical company, Safety

\section{Dear Editor,}

The first report of Coronavirus Disease 2019 (COVID-19) was presented in Wuhan, Hubei province, China, in December 2019 (1). COVID-19 is an infectious virus with a high level of spread. According to its fast pandemic condition in the world, it has been announced as an emergency of public health by the World Health Organization (WHO) related to its fast pandemic condition (1-3). Movements of population, communication among humans, and environmental factors cause the transfer of this virus. The most prevalent clinical symptoms include fever, cough, headache, muscle cramps, and fatigue (4). Different industries in different countries have faced many problems and even there were many days off in the world after the COVID-19 pandemic. The pharmaceutical industry, among all industries, has a unique and exclusive sensitivity according to the necessity in the production of pharmaceutical products. The pharmaceutical industry has been important since the past years as a complementary part of the primary process in the treatment of the patients that shows the significance of development in this industry.

In the COVID-19 pandemic situation, pharmaceutical companies try to respond to the challenges in the supply chain, change the business process, and protect the health of the staff. If the epidemic of COVID-19 continues for a medium/long time, this will affect active supplies, necessary materials, and medication export/import. Moreover, it causes adverse effects on research and development (R\&D) activities, production, and developmental projects related to improving the industry. Although the effect of global expansion has not been apparent yet, pharmaceutical companies should respond, improve, and develop. Therefore, during the COVID-19 pandemic situation, pharmaceutical companies should continue their activities and even develop it while facing many challenges. Moreover, it will help them detect the challenges and approaches of development in safety and health in pharmaceutical companies during the COVID-19 pandemic situation.

Challenges of pharmaceutical companies in the COVID-19 pandemic situation include: 1) Hiding or lack of reports in COVID-19 affection; 2) Lack of appropriate monitoring of distribution vehicles in the prevention of infection; 3) Continual contacts with documents and internal permissions of products leading to the lack of supervision of health principles; 4) Production in closed and limited space; 5) Continual production line and necessity of team working; 6) Commotion of the personnel with public transportation and lack of knowledge in family health; 7) The physical presence of staff in administrative positions (R\&D, marketing, IT, and planning); 8) Serving food in the restaurant of the company (breakfast, lunch, dinner, and snack); 9) Closing of air conditioning system in departments; 10) Lack of correct extrusion of produced wastes; 11) Lack of the attention of personnel on their health; and 12) The entrance of infected products and raw materials to the COVID-19.

Solutions in safety and health improvement of pharmaceutical company in facing COVID-19 pandemic include: 1) Disinfection of the external surface of the vehicles which contain materials and raw materials barrels at the entrance; 2) Use of electronic forms for documentation, product permissions, other production processes, quality control, and Health and Safety Executive ; 3) Use of distancing between the personnel with separator and fresh air injection into the rooms with building 
management system equipment; 4) Supply of mask and shield for all staff in sufficient number, and control of the use, extirpation, and protection of documents; 5) Use of masks with cartridge and N95 pad in administrative departments in exposure to chemical materials and changing them according to standard; 6) Assignment of the dedicated vehicles to the staff communication and their disinfection; 7) The necessity of using masks by the staff during transportation; 8) Provision of the opportunity for administrative staff to work from long distance; 9) Installation of the bags and buckets for sanitary wastes (mask, gloves, and tissue); 10) Disinfection of air conditioning system based on WHO rules; 11) Disinfection of all spaces and surfaces per hour or after use; 12) Installation of automatic disinfection equipment at the entrance of all buildings and busy places; 13) Introduction of COVID-19 as a job sickness to fast identification and self-declaration of the staff and elimination of transferring chain; 14) Assignment of subvention to treatment and leave of absence with salary for COVID-19 patients; 15) Psychological consultation and call contact with COVID-19 patients; 16) Online monitoring of the personnel and their families with an online questionnaire; 17) Transfer and management of waste by mechanizing systems and trained executive team with personal protection equipment; 18) Arrangement of all internal and external meetings online; 19) Presentation of all training courses in online classes (sky-room webinar); 20) Risk assessment in facing COVID-19 patients based on age, background illness, facing jobs, and a team of colleagues; 21) Non-public quarantine of the staff according to the importance of medicine production; 22) Specialization in COVID-19 tests for a suspicious person; 23) Distribution of selfprotect equipment and disinfectants among the staff's families; 24) Quarantine of suffering, suspicious people or those who were in contact with suffering patients; 25) Quarantine of the products in the warehouse to eliminate the transporting chain of the infected products.

\section{Conclusion}

The pharmaceutical stability of industry and permanent presence is an inseparable part of treatment teams in the world. Therefore, the necessity of continual observation of environmental health in pharmaceutical companies and the staff health could develop the efficiency, health protection of the personnel, and consumers in the COVID-19 pandemic situation. Controlling the challenges, as the next step, helps the presence of pharmaceutical companies in the current condition. Therefore, international, national, and local organizations should emphasize the revision of health and safety standards in the workplace. In addition, the self-declaration of industries and physical observation is necessary to conduct the suggested solutions for the personnel health as a staff in the health area.

\section{Footnotes}

Conflicts of Interest: The authors have no conflict of interest to declare.

Funding/Support: Nil.

Financial Disclosure: None declared.

\section{References}

1. Huang C, Wang Y, Li X, Ren L, Zhao J, Hu Y, et al. Clinical features of patients infected with 2019 novel coronavirus in Wuhan, China. Lancet. 2020;395(10223):497-506. doi: 10.1016/S01406736(20)30183-5. [PubMed: 31986264].

2. Zhu N, Zhang D, Wang W, Li X, Yang B, Song J, et al. A novel coronavirus from patients with pneumonia in China, 2019. $N$ Engl J Med. 2020;382(8):727-33. doi: 10.1056/NEJMoa2001017. [PubMed: 31978945].

3. Chen N, Zhou M, Dong X, Qu J, Gong F, Han Y, et al Epidemiological and clinical characteristics of 99 cases of 2019 novel coronavirus pneumonia in Wuhan, China: a descriptive study. Lancet. 2020;395(10223):507-13. doi: 10.1016/S01406736(20)30211-7. [PubMed: 32007143].

4. Wang W, Tang J, Wei F. Updated understanding of the outbreak of 2019 novel coronavirus (2019-nCoV) in Wuhan, China. J Med Virol. 2020;92(4):441-7. doi: 10.1002/jmv.25689. [PubMed: 31994742]. 